\title{
Future trends in nanotechnology aiming environmental applications
}

\author{
Leonardo Fernandes Fraceto ${ }^{1}$ Renata de Lima $^{2} \cdot$ Halley Caixeta Oliveira ${ }^{3}$. \\ Daiana Silva Ávila ${ }^{4} \cdot$ Bin Chen $^{5}$ \\ ${ }^{1}$ Department of Environmental Engineering, São Paulo State University (UNESP), Avenida Três de Março, no 511, Sorocaba, SP 18087-180, \\ Brazil \\ ${ }^{2}$ Department of Biotechnology, University of Sorocaba, Rodovia Raposo Tavares, Km 92.5, Sorocaba, SP 18023-000, Brazil \\ ${ }^{3}$ Department of Animal and Plant Biology, UEL-State University of Londrina, Rodovia Celso Garcia Cid, Km 380, Londrina, PR 86057-970, \\ Brazil \\ ${ }^{4}$ Pampa Federal University- UNIPAMPA, BR 472, Km 592, POBox 118, Uruguaiana, RS 97501-689, Brazil \\ ${ }^{5}$ State Key Joint Laboratory of Environment Simulation and Pollution Control, School of Environment, Beijing Normal University, Beijing, \\ China
}

Published online: 26 March 2018

(c) Joint Center on Global Change and Earth System Science of the University of Maryland and Beijing Normal University and Springer-Verlag GmbH Germany, part of Springer Nature 2018

Nanotechnology has become increasingly a reality nowadays, and along with it there is a need for discussions related to potential advances, as well as the impacts on the environment and human health that technology can cause (Purohit et al. 2017). Nanomaterials applications and also implications on the environment have been studied by several groups around the world, and particularly in Brazil, where nanotechnology has been increasingly applied on agriculture and their impacts are still unknown.

In this context, the II Workshop in Environmental Nanotechnology was an interdisciplinary forum of discussions about the aspects of this new technology, as well as, its innovation in the environmental area, contributing to the education of undergraduate and graduate students and also as an interchange and exchange of experiences among the invited speakers, professors, researchers and students of several post-graduation programs, contributing to the generation and spreading of knowledge in the

Leonardo Fernandes Fraceto

leonardo@sorocaba.unesp.br

Renata de Lima

renata.lima@prof.uniso.br

Halley Caixeta Oliveira

halley@uel.br

Daiana Silva Ávila

daianaavila@unipampa.edu.br

Bin Chen

chenb@bnu.edu.cn environmental nanotechnology area. The Workshop in Environmental Nanotechnology was held at the Institute of Science and Technology of São Paulo State University (UNESP) Campus Sorocaba in cooperation with researchers from other Brazilian Universities (University of Sorocaba, State University of Londrina, Federal University of Lavras, Federal University of Pampa, State University of Campinas). The aim of IIWEnNan special issue was to compile discussions regarding the aspects related to the environmental applications of Nanotechnology presented during the II Workshop in Environmental Nanotechnology, as well as the methods and techniques to the study of nanoparticles in the environment. Besides, it will approach some aspects related to the toxicity of the nanomaterial and its possible impacts on the environment. In this context, the present special issue will be a forum on spread of knowledge and improvement for researchers, professors and undergraduate and graduate students from this research field. In this special issue, we invited the participants from the Workshop to submit manuscripts in the following types: original full-length research articles, short communications, mini reviews, reviews, case studies, meta-analysis and perspective articles. 


\section{Thematic areas}

This special issue was targeted to participants of the II Workshop in Environmental Nanotechnology (IIWEnNan2016 2016) cover papers in the following areas:

- Sustainable nanotechnology

- Environmental applications of nanotechnology

- Agriculture applications

- Toxicity of nanomaterials

- Fate of pollutants in the environment

- Analytical methods aiming environmental applications

After the peer review process, we had accepted manuscripts in the following issues:

a. Cyclodextrins as effective tools to reduce the toxicity of atrazine

Among their diverse applications, nanomaterials have been used for decreasing the toxicity of agrochemicals to nontarget organisms (Grillo et al. 2016; Sadeghi et al. 2017). In addition to nano-based systems, others materials can be employed as molecular carriers for agrochemicals, such as the cyclic oligosaccharides cyclodextrins. The study of Venceslau et al. describes the development of a cyclodextrin-silica hybrid system incorporated with atrazine, an herbicide widely applied in the Americas with great contamination potential. The developed complex was shown to effectively decrease the toxicity of atrazine against lettuce (Lactuca sativa) seedlings, as demonstrated by the reduction of chromosomal aberrations and nuclear abnormalities in the bioassays in comparison with noncomplexed atrazine.

b. Soda lignin from Citrus sinensis bagasse: extraction, NMR characterization and application in bio-based synthesis of silver nanoparticles

There is an increasing concern regarding the sustainability of the methods used for the synthesis of nanomaterials. Instead of classical chemical methods, green syntheses of nanomaterials have emerged in recent years, with the use of plant extracts, fungi or bacteria in the procedures (Sharma et al. 2009). The study of Barros et al. reports the bio-based synthesis of silver nanoparticles using soda lignin extract from orange (Citrus sinensis) bagasse as a reducing and stabilizing agent. After full sequential extraction, the obtained soda lignin was characterized by nuclear magnetic resonance and then used for the successful production of highly stable silver nanoparticles. Thus, this study is a successful example of how a renewable material extracted from agroindustrial waste can have nanotechnological applications. c. Protein oxidation in the fish Danio rerio (Cyprinidae) fed with single- and multi-walled carbon nanotubes

The increasing development of nanotechnology is paralleled with the concern about the risk of environmental contamination with nanomaterials (Kah 2015; Sadeghi et al. 2017). Thus, research is necessary to address the potentially toxic effects of different types of nanomaterials on model organisms and to establish safety and regulatory protocols. Seixas et al. describe in vivo assays with Danio rerio (zebrafish) fed with single- and multi-walled carbon nanotubes, one of the nanomaterials with the greatest bioaccumulation potential. The data showed high levels of protein carbonylation in many fish organs in response to carbon nanotube exposition, demonstrating the induction of oxidative stress by this nanomaterial in an aquatic organism.

d. Nanotoxicology assessment in complementary/alternative models

The importance of nanotoxicological in vivo studies using alternative models other than mammalians is discussed by Avila et al., given that nanotoxicity occurs at different trophic levels. The minireview reports diverse nanotoxicological studies employing alternative models, such as the above-mentioned zebrafish, the nematode Caenorhabditis elegans, the Drosophila melanogaster, and the microcrustaceans Artemia salina and Daphnia magna. In this context, this topic is important to promote a discussion regarding the safety of nanomaterials to organisms that can be used to assess environment pollution and to predict effects in more complexes animals, as well as to the establishment of regulatory frameworks.

Notably, the special issue IIWEnNan2016-Workshop in Environmental Nanotechnology has selected key topic papers that give to the readers a good overview about the importance of the discussions in the field of environmental nanotechnology.

Acknowledgements The editors would like to thank the opportunity from E3 journal to host a special issue for the II Workshop in Environmental Nanotechnology. Also, the authors would like to thank for all support from the Editorial office and all reviewers that contribute to the peer review process. Also, the authors would like to thank the following agencies for the financial support to the workshop: CAPES and ACS.

\section{References}

Grillo R, Abhilash PC, Fraceto LF (2016) Nanotechnology applied to bioencapsulation of pesticides. J Nanosci Nanotechnol 16:1231-1234

IIWEnNan2016, in http://www.sorocaba.unesp.br/\#!/graduacao/ engenharia-ambiental/paginas-docentes/leonardo/workshop/. Accessed 24 Feb 2018 
Kah M (2015) Nanopesticides and nanofertilizers: emerging contaminants or opportunities for risk mitigation? Front Chem 3:64

Purohit R, Mittal A, Dalela S, Warudkar V, Purohit K, Purohit S (2017) Social, Environment and ethical impacts of nanotechnology. Mater Today 4:5461-5467
Sadeghi R, Rodriguez RJ, Yao Y, Kokini JL (2017) Advances in nanotechnology as they pertain to food and agriculture: benefits and risks. Annu Rev Food Sci Technol 8:467-492

Sharma V, Yngard R, Lin Y (2009) Silver nanoparticles: green synthesis and their antimicrobial activities. Adv Colloid Interface Sci 145:83-96 